\title{
Closed loop observers bundle for uncertain biotechnological models
}

Olivier BERNARD — Jean-Luc GOUZÉ

$\mathbf{N}^{\circ} 4905$

Août 2003

THÈME 4 



\title{
Closed loop observers bundle for uncertain biotechnological models
}

\author{
Olivier BERNARD, Jean-Luc GOUZÉ \\ Thème 4 - Simulation et optimisation \\ de systèmes complexes \\ Projet Comore \\ Rapport de recherche $n^{\circ} 4905$ - Août 2003 - 22 pages
}

\begin{abstract}
We propose nonlinear observers for a class of biotechnological processes. These observers are an extension of the asymptotic observers (observers with unknown inputs) devoted to biotechnological systems for which some parts of the model are unknown. We take benefit of the additional outputs which are (non linear) functions of the state to design a closed loop observer. The global convergence of these nonlinear observers is proven. We use then these observers to design interval based observers which predict the intervals in which the state is lying. We run in parallel a broad set of interval observers and we select the best ones. The method is illustrated with a model describing the bioconversion of a substrate using microorganisms in a bioreactor.
\end{abstract}

Key-words: nonlinear observers, unknown inputs, mass balance models, bioprocesses, robustness, bundle of observers. 


\section{Multi-observateurs pour des modèles biotechnologiques mal connus}

Résumé : Nous construisons des observateurs pour des procédés biotechnologiques. Ce sont des extensions des observateurs asymptotiques (en boucle ouverte), qui permettent d'estimer l'état de modèles dont certaines parties sont inconnues. Nous utilisons des sorties supplémentaires pour construire des observateurs nonlinéaires en boucle fermée. Puis nous contruisons des observateurs similaires, mais à intervalles, qui donnent une estimation garantie. Cela nous permet de lancer un ensemble d'observateurs simultanéement, pour améliorer les performances. Nous donnons l'exemple d'un modèle décrivant la bioconversion d'un substrat par des microorganismes en bioréacteur.

Mots-clés : observateurs non linéaires, entrées inconnues, bioprocédés, multi observateurs. 


\title{
Closed loop observers bundle for uncertain biotechnological models
}

\author{
Olivier BERNARD, Jean-Luc GOUZÉ \\ COMORE, INRIA, \\ BP 93, 06902 Sophia-Antipolis Cedex, France \\ Fax: +33492387858 \\ E-mails: olivier.bernard@inria.fr, jean-luc.gouze@inria.fr
}

\section{Introduction}

The biotechnological processes in stirred tank reactors can be modeled by nonlinear differential equations involving nonlinear biological kinetics (e.g. cellular growth rate, bioproduction rate, ...). Very often, the precise formulation of these kinetics is not well known, and rough approximations and hypotheses are made to write the model. Let us suppose now that we are able to measure without noise some outputs, and that we want to build an observer to estimate the state variables. Some rather general methodologies exist for nonlinear systems $[9,8]$ and in particular for biological system [5], but the model has to be exactly known. Because of the bad quality of the models in biotechnology, it is often preferable to build a different kind of observer (often called asymptotic observer), not using the unknown parts (biological kinetics) of the model. In the counterpart these observers have weaker properties concerning, for example, the rate of convergence $[1,2]$. These observers [1] are based on mass-balance considerations, and since they are in open-loop, they are not very robust to errors on the estimation of the influent masses. Nevertheless these observers require the measurements of some gaseous flow rates. In this paper we take benefit of these outputs and of other measurements rather easy to perform in practice ( $\mathrm{pH}$, conductivity, ...). These outputs rest on physical or chemical laws that are well known, and provide therefore reliable (nonlinear) relationships with the state variables. Following the idea presented in [4], we use these outputs to build a closed-loop nonlinear observer, that does not use the unknown kinetics, but takes advantage of the additional outputs to achieve better properties of robustness and convergence rate.

This problem is related with the linear theory of rejection of unknown inputs $[13,12,7]$, but we are here in a nonlinear framework. Moreover, we use some specificities of the biological models that we consider. In particular, the classes of system that we consider (where the kinetics are considered as unknown inputs) are generally not observable (but detectable), so that the classical methods for observers with unknown inputs cannot be applied.

Then we improve the proposed observers by using their properties linked to differential inequalities and positivity to design interval observers $[11,10]$ that can deal with uncertainties, such as imprecision in the 
influent concentration measurement. The idea of interval observers is to propose an estimate of the interval in which the state is sure to lie.

A new trend in the design of observers is the idea to initialize simultaneously several observers and to take benefit of all these estimates in order to improve the state estimation [6]. We exploit this idea here and the tuning possibility of the observers which all guarantee a bounding of the real trajectories. Because of the interval framework, we are able to take the best smallest envelope of this bundle of observers, to improve the estimation in a striking way.

The paper is organized as follows: after some definitions, we recall the notion of "asymptotic observers" in open loop (section 2), then give a closed loop observer ( section 3) and show later that this observer is more robust to errors in the inputs (section 4). Then we show how to design an interval observer which estimates bounds for the state variables (section 5). Combining a set of such observers, we show how to improve the state estimate. We end up in section 6 by an example where a model of bioprocess is simulated and a bundle of observers is proposed for this model and compared with the more classical open loop interval observer.

\section{Notations and definitions}

A bioprocess in a stirred tank bioreactor can be described by a set of $k$ coupled micro-biological and biochemical reactions which take place in the reactor and involve a set of $n$ biological or chemical species such as micro-organisms, substrates, metabolites, enzymes...

The dynamical behavior of a continuously stirred tank bioreactor (CSTR) is often described by a general mass-balance model of the following form (see e.g. [1, 2]):

$$
\dot{\xi}=K r(\xi)-D(t) \xi+D(t) \xi_{\text {in }}(t)-Q(\xi)
$$

In this model, the vector $\xi=\left(\xi_{1}, \xi_{2}, \ldots, \xi_{n}\right)^{t}$ is the vector of the concentrations of the various species inside the liquid medium. The first term $K r(\xi)$ on the right-hand side represents the biological and biochemical conversions in the reactor (per unit of time) according to the underlying reaction network. The $(n \times k)$ matrix $K$ is a constant (stoichiometric-like) yield coefficient matrix. The vector $r(\xi)=\left(r_{1}(\xi), r_{2}(\xi), \ldots, r_{k}(\xi)\right)^{t}$ is a vector of reaction rates (or conversion rates). The influent feeding concentration is represented by $\xi_{\text {in }}(t)$, and $D(t)$, the dilution rate is the ratio of the influent flow rate over the volume of the fermenter. For sake of brevity the time dependence will be omitted in the future. The last term $Q(\xi)$ represents the gaseous exchange with the outside of the fermenter.

In the sequel the mappings $r(\xi), Q(\xi)$ are assumed to be $\mathcal{C}^{1}$ on the considered nonnegative domain $\Omega$. The solutions of the ordinary differential equations are well defined and unique.

Hypothesis 1 (H1) We assume that the trajectories of system (1) are (lower and upper) bounded on $\Omega$ for given bounded inputs $\xi_{\text {in }}$ and $D$ and given positive initial conditions.

This property is generally verified for mass balance systems [3].

We assume that a part $\xi_{1}$ of the state $\xi$ can be measured on-line. The part of the state which is not measured, $\xi_{2}$, is of dimension $p$. We have thus $\xi=\left(\xi_{1}, \xi_{2}\right)^{t}$. We assume moreover that the output $y$ of the 
system (i.e. the set of measurements) can be split into 3 parts:

$$
y=\left(\begin{array}{l}
y_{1} \\
y_{2} \\
y_{3}
\end{array}\right)
$$

with:

$$
\begin{aligned}
& y_{1}=\xi_{1} \in \mathbf{R}^{n-p} \\
& y_{2}=Q(\xi) \in \mathbf{R}^{n} \\
& y_{3}=h\left(\xi_{1}, \xi_{2}\right) \in \mathbf{R}^{m}
\end{aligned}
$$

In other words we suppose that we can measure the gaseous flow rates $Q(\xi)$ and also a set of functions of the state $h\left(\xi_{1}, \xi_{2}\right)$. They can be physical variables ( $\mathrm{pH}$, partial pressure of a gas, etc.) related to the state $\xi$. As it will be illustrated in the following, in the general case there does not exist any injective relationship between $y$ and $\xi$. Indeed the vectors $y_{2}$ and $y_{3}$ are, in many realistic cases, mainly composed of zeros, and the non-zero terms can be complicated and non-injective expressions of the whole state. We have therefore to estimate the variables with an observer.

Example. Along the paper we will illustrate our method with a very simple biotechnological example. We assume that a bacterial biomass $(X)$ is growing in a CSTR. The micro-organisms consume the substrate $S$ and produce a product $P$. As most of the time in biotechnology, the bacterial growth rate $r(\xi)$ is a complex function of the state which is generally poorly known. In the sequel we assume that $r(\xi)$ is unknown.

The model associated with this example is thus the following:

$$
\left\{\begin{array}{l}
\dot{X}=r(\xi)-D X \\
\dot{S}=-c_{1} r(\xi)+D\left(S_{i n}-S\right) \\
\dot{P}=c_{2} r(\xi)+D\left(P_{\text {in }}-P\right)
\end{array}\right.
$$

where $S_{i n}$ and $P_{i n}$ are the influent substrate and product concentrations, and $c_{1}$ and $c_{2}$ are yield coefficients. This model has the form presented in (1) with:

$$
K=\left(1,-c_{1}, c_{2}\right)^{t}, \xi_{\text {in }}=\left(0, S_{\text {in }}, P_{\text {in }}\right)^{t}, Q(\xi)=(0,0,0)^{t}
$$

We assume that the bacterial biomass can be measured. Moreover we suppose that we can estimate the proportion of $S$ in the medium with respect to $P$. These relative measurements are often much easier (and also less expensive) to obtain than the absolute values.

We have therefore:

$$
\begin{aligned}
& y_{1}=X \\
& y_{2}=(0,0,0)^{t} \\
& y_{3}=\frac{S}{S+P}
\end{aligned}
$$

Our aim is to estimate $S$ and $P$, without using the unknown kinetics $r(\xi)$. 
This example is generic, and in most of the cases $S$ could be the oxygen and $P$ the $\mathrm{CO}_{2}$. Then the gaseous exchanges with atmosphere have to be taken into account.

Here we will consider the anoxic denitrification by denitrifying bacteria. Then the substrate $\mathrm{S}$ corresponds to nitrate and the product $\mathrm{P}$ to nitrite. The influent is a blend of nitrate and nitrite.

Instead of measuring the $\mathrm{NO}_{3}$ and $\mathrm{NO}_{2}$ concentration, it is simpler to measure the ratio $\frac{\mathrm{NO}_{2}}{\mathrm{NO}_{3}}$ even if so far the usual colorimetric sensors also provide the estimate of the concentrations after a calibration step. One consequence of our study could be to stimulate the development of these simpler qualitative sensors which provide an accurate information after coupling with an observer.

\subsection{Recall: the asymptotic observers}

For details the reader may consult [1]. We separate the measured state variables $\xi_{1}$ and the unmeasured state variables $\xi_{2}$. We rewrite system (1) as follows:

$$
\begin{aligned}
& \dot{\xi}_{1}=K_{1} r(\xi)-D \xi_{1}+D \xi_{i n 1}-Q_{1}(\xi) \\
& \dot{\xi}_{2}=K_{2} r(\xi)-D \xi_{2}+D \xi_{i n 2}-Q_{2}(\xi)
\end{aligned}
$$

where $\left(K_{1}, K_{2}\right),\left(\xi_{i n 1}, \xi_{i n 2}\right)$ and $\left(Q_{1}(\xi), Q_{2}(\xi)\right)$ are the induced partition of $K, \xi_{\text {in }}$ and $Q(\xi)$ respectively.

We state now an hypothesis on the rank of the matrix $K_{1}$ :

Hypothesis 2 (H2) The $(n-p) \times k($ with $(n-p) \geq k)$ matrix $K_{1}$ has full rank.

From (H2), since it has full rank, $K_{1}$ admits a left inverse P:

$$
P K_{1}=I_{k}
$$

where $I_{k}$ denotes the identity matrix of $\mathbf{R}^{k}$. Now if we denote

$$
A \stackrel{\text { def }}{=}-K_{2} P
$$

we consider then the following linear change of coordinates:

$$
\begin{aligned}
\zeta_{1} & =\xi_{1} \\
\zeta_{2} & =A \xi_{1}+\xi_{2}
\end{aligned}
$$

this change of variables transforms (11) and (12) into:

$$
\begin{aligned}
& \dot{\zeta}_{1}=K_{1} r(T \zeta)-D \zeta_{1}+D \zeta_{i n 1}-Q_{1}(T \zeta) \\
& \dot{\zeta}_{2}=-D\left(\zeta_{2}-\zeta_{i n 2}\right)-\left(A Q_{1}(T \zeta)+Q_{2}(T \zeta)\right)
\end{aligned}
$$

with

$$
T \stackrel{\text { def }}{=}\left(\begin{array}{cc}
I_{n-p} & 0_{n-p, p} \\
-A & I_{p}
\end{array}\right), M \stackrel{\text { def }}{=}\left(\begin{array}{ll}
A & I_{p}
\end{array}\right)
$$

and

$$
\zeta_{\text {in } 2}=M \xi_{\text {in }}
$$


The equation of 18 can be rewritten using the output $y_{2}$ :

$$
\dot{\zeta}_{2}=-D\left(\zeta_{2}-\zeta_{i n 2}\right)-M y_{2}
$$

Remark: It is worth noting that system (21) is a linear system up to an output injection.

Hypothesis 3 (H3) The positive scalar input $D(t)$ is persistently exciting i.e. there exists positive constants $\alpha$ and $\epsilon$ such that

$$
0<\alpha \leq \int_{t}^{t+\epsilon} D(\tau) d \tau
$$

For sake of simplicity we will assume in the proof that the dilution rate $D$ is a positive constant. However all the results apply with a varying $D(t)$ satisfying (H3).

Lemma 1 [1] Under hypothesis (H3), the solution $\hat{\xi}_{2}$ of the following open-loop observer:

$$
\begin{aligned}
& \dot{\hat{\zeta}}_{2}=-D\left(\hat{\zeta}_{2}-\zeta_{i n 2}\right)-M y_{2} \\
& \hat{\xi}_{2}=\hat{\zeta}_{2}-A y_{1}
\end{aligned}
$$

converges asymptotically toward the solution $\xi_{2}$ of the reduced system (12).

Proof: It is easy to verify that the estimation error $e_{2} \stackrel{\text { def }}{=} \hat{\xi}_{2}-\xi_{2}=\hat{\zeta}_{2}-\zeta_{2}$ satisfies:

$$
\dot{e}_{2}=-D e_{2}
$$

and converges asymptotically toward $\xi_{2}$.

Remark: It is worth noting that the linear system (21) is not observable for any linear output $y=C \zeta$. Indeed it is a set of $p$ decoupled first order equations.

Example: for the above mentioned example we have (with $\xi_{1}=X, \xi_{2}=(S, P)$ ):

$$
T=\left(\begin{array}{ccc}
1 & 0 & 0 \\
c_{1} & 1 & 0 \\
-c_{2} & 0 & 1
\end{array}\right)
$$

leading to the following open loop observer:

$$
\begin{aligned}
\dot{\hat{z}}_{1} & =-D\left(\hat{z}_{1}-z_{i n 1}\right) \\
\dot{\hat{z}}_{2} & =-D\left(\hat{z}_{2}-z_{i n 2}\right) \\
\hat{S} & =\hat{z}_{1}-\frac{y_{1}}{c_{1}} \\
\hat{P} & =\hat{z}_{2}+\frac{y_{1}}{c_{2}}
\end{aligned}
$$

with $z_{i n 1}=S_{i n}$ and $z_{i n 2}=P_{i n}$.

This observer has nevertheless several defects. It is in open loop and therefore it is assumed that the model (except the unknown reaction rates) is perfectly known and that there is no error in the measurements, in the estimations of the feeding inputs and in the estimates of the yield coefficient matrix $K$. Moreover this asymptotic observer does not take benefit of all the available information $\left(y_{2}\right.$ and $\left.y_{3}\right)$.

$\mathrm{RR} \mathrm{n}^{\circ} 4905$ 


\section{Closed loop mass balance observers}

\subsection{Motivation}

The asymptotic observers are based on mass balance considerations, but in some cases the exact mass flow rate $\xi_{\text {in }}$ entering in the system is not well known. This is for example the case for waste water treatment when the exact mass of polluting substrate in the influent is not measured. In that case of corrupted information on the system inputs, the predictions of the mass balance will be biased. It is worth noting that, due to their specific constructions, these observers are in open loop. Indeed the predictions of these observers are not compared with any measurements in order to correct the estimations. In the following we will show that such a correction is possible when an online measurement is a function of the state variables. This is for example the case for the gas flow rates which are generally a linear function of the concentration of the compounds in the dissolved phase. Many other additional (linear or nonlinear) outputs are possible.

\subsection{Principle}

We suppose here that $y_{3} \in \mathbf{R}$ : the mapping $h$ is thus defined as follows:

$$
h:\left(\xi_{1}, \xi_{2}\right) \in\left(\mathbf{R}^{n-p} \times \mathbf{R}^{p}\right) \longrightarrow y_{3}=h\left(\xi_{1}, \xi_{2}\right) \in \mathbf{R}
$$

We assume that $h$ satisfies the following hypotheses (by convention, $\frac{\partial h}{\partial \xi_{2}}$ is a line vector):

Hypothesis 4 (H4) The mapping $h$ is monotone with respect to $\xi_{2}$, i.e. $\frac{\partial h}{\partial \xi_{2}}$ is of fixed signs on $\Omega$.

Example: in our example, $\xi_{1}=X$ and $\xi_{2}=(S, P)$, then $h(X, S, P)=\frac{S}{S+P}$, and thus:

$$
\frac{\partial h}{\partial \xi_{2}}=\left[\frac{P}{(S+P)^{2}}-\frac{S}{(S+P)^{2}}\right]
$$

which is of fixed signs on $\mathbf{R}_{+}^{2}$.

Change of notations: To lighten the notation, we will denote $z=\zeta_{2}$, so that the real system satisfies:

$$
\begin{aligned}
& \dot{z}=-D\left(z-z_{i n}\right)-M y_{2} \\
& \xi_{2}=z-A y_{1}
\end{aligned}
$$

Proposition 1 Let $\lambda \in \mathbf{R}^{p}$ be a constant unit vector $(\|\lambda\|=1)$, whose signs are chosen such that $\lambda_{i} \frac{\partial h}{\partial \xi_{2 i}} \geq 0$ (for $1 \leq i \leq p$ ), $\theta$ is a (possibly time varying) positive scalar, and $z_{i n}=M \xi_{i n}$. The following system

$$
\dot{\hat{z}}=-D\left(\hat{z}-z_{i n}\right)-M y_{2}-\theta \lambda\left(h\left(y_{1}, \hat{z}-A y_{1}\right)-y_{3}\right)
$$

is an observer of the reduced system (30).

Remark: the inequality $\lambda_{i} \frac{\partial h}{\partial \xi_{2 i}} \geq 0$ has to be satisfied for the state $\xi$, but also for the estimated state $\left(\xi_{1}, \hat{\xi}_{2}\right)$. As a consequence, it must be checked whether $h\left(y_{1}, \hat{\xi}_{2}\right)$ remains monotone when $\hat{\xi}_{2}$ is outside $\Omega$. If this property does not hold, $h$ must be prolongated in the negative domain to satisfy the good properties. This is illustrated in our final example.

To prove this result, we first show the following Lemma. 
Lemma 2 If the trajectories of system (1) are bounded then the trajectories of the observer given by system (31) are bounded.

Proof of the Lemma: The equation error is (with $e \stackrel{\text { def }}{=} \hat{z}-z$ ):

$$
\dot{e}=-D e-\theta \lambda\left(h\left(y_{1}, \hat{z}-A y_{1}\right)-h\left(y_{1}, z-A y_{1}\right)\right)
$$

We consider $l_{1}, \ldots, l_{p-1} \in \mathbf{R}^{p}$ an orthonormal basis of the vectorial subspace orthogonal to $\lambda$.

Let us denote by $\tilde{e}$ the error in the new basis given by $\left(l_{1}, \ldots, l_{p-1}, \lambda\right)$.

The components along $l_{i}$, the $i^{\text {th }}$ vector $(i=1, \ldots, p-1)$ of the basis is given by

$$
\tilde{e}_{i}=l_{i}^{t} e
$$

Since the $l_{i}$ are orthogonal to $\lambda$, it is clear from (32) that:

$$
\dot{\tilde{e}}_{i}=-D \tilde{e}_{i}
$$

It becomes then clear that the $(p-1)$ first components are bounded:

$$
\min \left(0, \tilde{e}_{i}(0)\right) \leq \tilde{e}_{i} \leq \max \left(0, \tilde{e}_{i}(0)\right.
$$

Now for the component $\tilde{e}_{p}$ associated to $\lambda\left(\tilde{e}_{p}=\lambda^{t} e\right)$ :

$$
\dot{\tilde{e}}_{p}=-D \tilde{e}_{p}-\theta\left(h\left(y_{1}, \hat{z}-A y_{1}\right)-h\left(y_{1}, z-A y_{1}\right)\right)
$$

Let us remind that the original system (1) is bounded (H1); it follows that $z-A y_{1}$ is bounded and since $h$ is continuous with respect to $\xi_{2}$, there exists $B_{m}$ and $B_{M}$ in $\mathbf{R}^{p}$ (depending on the initial conditions in $z$ and $\hat{z}$ ) such that:

$$
\left.h\left(y_{1}, B_{m}\right) \leq h\left(y_{1}, z-A y_{1}\right)\right) \leq h\left(y_{1}, B_{M}\right)
$$

In the same way, there exists two bounds $C_{m}$ and $C_{M}$ in $\mathbf{R}^{p}$ such that the term $h\left(y_{1}, \hat{z}-A y_{1}\right)=$ $h\left(y_{1}, z+e-A y_{1}\right)$ can be bounded:

$$
h\left(y_{1}, C_{m}+B_{m}+\lambda \tilde{e}_{p}\right) \leq h\left(y_{1}, z-A y_{1}+\sum_{i=1}^{p-1} l_{i} \tilde{e}_{i}+\lambda \tilde{e}_{p}\right) \leq h\left(y_{1}, C_{M}+B_{M}+\lambda \tilde{e}_{p}\right)
$$

We have then

$$
-D \tilde{e}_{p}+\theta\left(h\left(y_{1}, C_{m}+B_{m}+\lambda \tilde{e}_{p}\right)-h\left(y_{1}, B_{M}\right)\right) \leq \dot{\tilde{e}}_{p} \leq-D \tilde{e}_{p}+\theta\left(h\left(y_{1}, C_{M}+B_{M}+\lambda \tilde{e}_{p}\right)-h\left(y_{1}, B_{m}\right)\right)
$$

We rewrite the term $h\left(y_{1}, C_{m}+B_{m}+\lambda \tilde{e}_{p}\right)-h\left(y_{1}, C_{m}+B_{m}\right)$ using a generalized Taylor formula:

$$
h\left(y_{1}, C_{m}+B_{m}+\lambda \tilde{e}_{p}\right)-h\left(y_{1}, C_{m}+B_{m}\right)=-\phi\left(C_{m}+B_{m}, \lambda \tilde{e}_{p}, y_{1}\right) \lambda \tilde{e}_{p}
$$

with $\phi\left(C_{m}+B_{m}, \lambda \tilde{e}_{p}, y_{1}\right)=\int_{0}^{1} \frac{\partial h}{\partial \xi_{2}}\left(y_{1}, C_{m}+B_{m}+\lambda \tau \tilde{e}_{p}\right) d \tau$. The same expression holds for the upper bounds; it leads us to the following expression:

$$
\begin{array}{r}
-\left(D+\theta \phi\left(C_{m}+B_{m}, \tilde{e}_{p}, y_{1}\right) \lambda\right) \tilde{e}_{p}+\theta\left(h\left(y_{1}, C_{m}+B_{m}\right)-h\left(y_{1}, B_{M}\right)\right) \\
\leq \dot{\tilde{e}}_{p} \leq \\
-\left(D+\theta \phi\left(C_{M}+B_{M}, \tilde{e}_{p}, y_{1}\right) \lambda\right) \tilde{e}_{p}+\theta\left(h\left(y_{1}, C_{M}+B_{M}\right)-h\left(y_{1}, B_{m}\right)\right)
\end{array}
$$

$\mathrm{RR} \mathrm{n}^{\circ} 4905$ 
Since we have chosen a $\lambda$ such that $\lambda_{i} \frac{\partial h}{\partial \xi_{2 i}} \geq 0$, it implies, by integration, that $\phi\left(C_{m}+B_{m}, \tilde{e}_{p}, y_{1}\right) \lambda \geq 0$ and $\phi\left(C_{M}+B_{M}, \tilde{e}_{p}, y_{1}\right) \lambda \geq 0$, and ensures the boundedness of $\tilde{e}_{p}$.

Proof of Proposition: Now the proof of the proposition follows. First, let us rewrite the equation error (33) for the component $\tilde{e}_{p}$, when $\tilde{e}_{i}=0$ for $i<p$. Using again the generalized Taylor formula:

$$
\dot{\tilde{e}}_{p}=-\left(D+\theta \phi\left(\hat{z}, z, y_{1}\right) \lambda\right) \tilde{e}_{p}
$$

with $\phi\left(\hat{z}, z, y_{1}\right)=\int_{0}^{1} \frac{\partial h}{\partial \xi_{2}}\left(y_{1}, \tau \hat{z}+(1-\tau) z-A y_{1}\right) d \tau$.

Since $\phi\left(\hat{z}, z, y_{1}\right) \lambda \geq 0$, it proves that the error along $\lambda$ converges globally exponentially with a rate larger or equal to $D$.

System (32) can now be rewritten as follows ( $v$ denotes the $(p-1)$ first $\tilde{e}_{i}, u=\tilde{e}_{p}$ ):

$$
(\Sigma)\left\{\begin{array}{l}
\dot{v}=g(t, v) \\
\dot{u}=f(t, u, v)
\end{array}\right.
$$

where $v=0$ is a globally exponentially stable equilibrium of $\dot{v}=g(t, v)$, and $u=0$ is a globally asymptotically stable fixed point for $\dot{u}=f(t, u, 0)$. We have proved the boundedness of the trajectories of (32), and thus, from [16], 0 is a globally asymptotically stable fixed point for system (32).

Proposition 2 Suppose moreover that $\phi \lambda$ is lower bounded by a positive real $\eta_{\text {min }}$ :

$$
0<\eta_{\min } \leq \phi\left(\hat{z}, z, y_{1}\right) \lambda, \forall\left(\hat{z}, z, y_{1}\right)
$$

then the convergence rate of $\tilde{e}_{p}$ is greater than $\left(D+\theta \eta_{\min }\right)$. It can be adjusted using $\theta$.

Example: We design the closed loop mass balance observer for the considered biotechnological process:

$$
\left\{\begin{array}{l}
\dot{\hat{z}}_{1}=D\left(z_{i n 1}-\hat{z}_{1}\right)-\theta \lambda_{1}\left(\frac{\hat{S}}{\hat{S}+\hat{P}}-y_{3}\right) \\
\dot{z}_{2}=D\left(z_{i n 2}-\hat{z}_{2}\right)-\theta \lambda_{2}\left(\frac{\hat{S}}{\hat{S}+\hat{P}}-y_{3}\right) \\
\hat{S}=\hat{z}_{1}-\frac{y_{1}}{c_{1}} \\
\hat{P}=\hat{z}_{2}+\frac{y_{1}}{c_{2}}
\end{array}\right.
$$

The convergence of this observer is a consequence of Proposition 1 if we choose $\lambda_{1}>0$ (same sign as $\frac{P}{(S+P)^{2}}$ ) and $\lambda_{2}<0$ (same sign as $-\frac{S}{(S+P)^{2}}$ ).

Remark: the same remark as the one after the Proposition 1 can be made here: outside the nonnegative domain, the function $h$ has to be prolongated.

\section{Robustness of the closed loop mass balance observer}

Before all, note that the closed loop correction does not accelerate the convergence rate of the observer, except in one direction. This can be easily seen on the equation (40). This is the consequence of the non observability of system (21); we cannot expect to tune freely the convergence rate.

In fact $\lambda$ is also a robustification parameter vector. In the following we will see how the closed loop system improves the errors on the estimates due to the modeling errors such as a bad estimation of the influent concentration. 
Let us examine the steady state predictions of the observer given by (22) when the information on the influent concentration is biased. We will consider in the following the case when the inputs of the system (dilution rate and influent concentrations) are asymptotically constant. Let us denote $e_{i n} \stackrel{\text { def }}{=} \tilde{z}_{i n}-z_{i n}$ the error between the assumed influent concentration $\left(\tilde{z}_{i n}\right)$ and the actual one $\left(z_{i n}\right)$. The error between $\hat{z}$ and $z$ at infinity is denoted $e_{\infty}$.

The equation of the open loop observer (22) show directly that, at equilibrium:

$$
e_{\infty}=e_{i n}
$$

The error on the state is exactly the error on the mass balance.

We will show that this error is reduced for the closed loop observer.

Note first that the problem at steady state is a linear problem since $\phi_{\infty}=\lim _{t \rightarrow+\infty} \frac{D h}{D \xi_{2}}$ can be computed (using the convergence of the observer given by Proposition 1):

$$
\phi_{\infty}=\frac{\partial h}{\partial \xi_{2}}\left(y_{1}, \hat{z}_{\infty}-A y_{1}\right)
$$

where $\hat{z}_{\infty}=\lim _{t \rightarrow+\infty} \hat{z}=\lim _{t \rightarrow+\infty} z$.

We consider now that the influent concentrations are biased and we will compute the steady state error $e_{\infty}$. We still assume that $\phi_{\infty}$ is known which is indeed a first order approximation. Note that it is exactly known if $h$ is linear.

First, it follows from (31) that the equation error is given by:

$$
\left(D I_{p}+\theta \lambda \phi_{\infty}\right) e_{\infty}=\delta
$$

where $\delta$ designs the modeling error vector. If the only unknown is the influent $e_{i n}$ then $\delta=D e_{i n}$.

Proposition 3 If we choose

$$
\lambda=\frac{\phi_{\infty}^{t}}{\left\|\phi_{\infty}\right\|}
$$

then, for any positive gain $\theta$, the error due to $\delta$ is reduced by the robust asymptotic observer:

$$
\left\|e_{\infty}\right\| \leq\left\|e_{\infty}^{0}\right\|
$$

where $e_{\infty}^{0}=\frac{\delta}{D}$ is the error obtained by the asymptotic observer in open loop (the norm $\|\cdot\|$ is the Euclidean norm).

proof: Note first that $\lambda \phi_{\infty}$ is a rank one matrix. With our choice of $\lambda=\frac{\phi_{\infty}^{t}}{\left\|\phi_{\infty}\right\|}$, it is a symmetric matrix. We can diagonalize it in an orthonormal base.

The non trivial eigenvalue is $\phi_{\infty} \lambda>0$ associated with the (unitary) eigenvector $\lambda$, indeed:

$$
\lambda \phi_{\infty} \lambda=\left(\phi_{\infty} \lambda\right) \lambda
$$

It is easy to see that there exists an orthogonal matrix $P$ such that

$$
P^{t}\left(D I_{p}+\theta \lambda \phi_{\infty}\right) P \tilde{e}_{\infty}=\tilde{\delta}
$$


giving in the new basis the equation (the tilde denotes the new basis):

$$
\Gamma \tilde{e}_{\infty}=\tilde{\delta}
$$

with:

$$
\Gamma=\left(\begin{array}{ccccc}
D+\theta \phi_{\infty} \lambda & 0 & \ldots & & 0 \\
0 & D & \ddots & & \\
& \ddots & \ddots & & \vdots \\
\vdots & & & D & 0 \\
0 & \ldots & & 0 & D
\end{array}\right)
$$

therefore

$$
\tilde{e}_{\infty}=\Gamma^{-1} \tilde{\delta}
$$

This proves that:

$$
\left\|e_{\infty}\right\|=\left\|\tilde{e}_{\infty}\right\|<\frac{\|\tilde{\delta}\|}{D}=\frac{\|\delta\|}{D}
$$

Remark: the first component of $\tilde{e}_{\infty}$ is decreased by a rate factor $\frac{D}{D+\theta \phi_{\infty} \lambda}$. If $\theta$ is large, this factor can be made as small as desired. This component is associated to the direction $\lambda$. Moreover, if more information is available on the influent error, other choices for $\lambda$ can be done.

\section{$5 \quad$ Interval observers and bundle of observers}

As we have seen above, it is very frequent that the influent concentrations, i.e. the $\xi_{i n}$, are not exactly known. We have seen that the closed loop observer improves the robustness of the open loop observer by providing a better asymptotic error.

We will suppose in this section that we know upper and lower bounds on the inputs, and we build interval observers, giving guaranteed bounds on the estimated state (an introduction to interval observer is given in [10], a more elaborated presentation is in [14]). Our first aim is to obtain bounds on the estimates ; our second aim is to build not only one observer, but a whole set of observers (called a bundle of observers), to be able to take the intersection of the bounds of each observer, and so to improve simultaneously the convergence rate and the asymptotic bounds on the estimates.

\subsection{Interval observer}

We suppose that we know the upper and lower bounds on the inputs, so that we can write:

$$
z_{\text {in }}^{-}(t) \leq z_{\text {in }}(t) \leq z_{\text {in }}^{+}(t)
$$

To simplify the presentation, we consider the case with $z=\left(z_{1}, z_{2}\right)$ in dimension two, but the result and the proofs are the same in dimension $p$. We also simplify the notation $h\left(y_{1}, z-A y_{1}\right)=h(z)$, and take $y_{2}=0$ (remember that $y_{2}$ disappears anyway when we compute the equation error). For sake of clarity, the function 
$h$ is supposed to be increasing with respect to the first component of $z$ and decreasing with respect to the second one, as in the example. The vector $\lambda$ has signs as in proposition 1 , therefore $\lambda_{1} \geq 0, \lambda_{2} \leq 0$.

Proposition 4 The following system is an interval observer for the variables $z(t)$ solution of (30)

$$
\begin{aligned}
& \dot{z}_{1}^{+}=D z_{i n 1}^{+}(t)-D z_{1}^{+}-\theta \lambda_{1}\left(h\left(z_{1}^{+}, z_{2}^{+}\right)-y_{3}\right) \\
& \dot{z}_{2}^{+}=D z_{i n 2}^{+}(t)-D z_{2}^{+}-\theta \lambda_{2}\left(h\left(z_{1}^{+}, z_{2}^{+}\right)-y_{3}\right) \\
& \dot{z}_{1}^{-}=D z_{i n 1}^{-}(t)-D z_{1}^{-}-\theta \lambda_{1}\left(h\left(z_{1}^{-}, z_{2}^{-}\right)-y_{3}\right) \\
& \dot{z}_{2}^{-}=D z_{i n 2}^{-}(t)-D z_{2}^{-}-\theta \lambda_{2}\left(h\left(z_{1}^{-}, z_{2}^{-}\right)-y_{3}\right)
\end{aligned}
$$

i.e., if at time zero

$$
z^{-}(0) \leq z(0) \leq z^{+}(0)
$$

then for positive time

$$
z^{-}(t) \leq z(t) \leq z^{+}(t)
$$

(the inequalities being taken on each component).

Proof: We start such that $z^{-}(0) \leq z(0) \leq z^{+}(0)$. If, for some time $t_{0}$, these inequalities are not true anymore, it implies that at least one of them is an equality. Let us suppose that, for example, $z_{1}\left(t_{0}\right)=z_{1}^{+}\left(t_{0}\right)$, then we calculate:

$$
\dot{z}_{1}^{+}-\dot{z}_{1}=D\left(z_{i n 1}^{+}\left(t_{0}\right)-z_{i n 1}\left(t_{0}\right)\right)-\theta \lambda_{1}\left(h\left(z_{1}^{+}, z_{2}^{+}\right)-h\left(z_{1}, z_{2}\right)\right)
$$

This derivative $\dot{z}_{1}^{+}-\dot{z}_{1}$ is made of two terms: the first is positive because of the bounds on the inputs, and the second one is such that, at time $t_{0}$ :

$$
h\left(z_{1}^{+}, z_{2}^{+}\right)-h\left(z_{1}, z_{2}\right)=h\left(z_{1}^{+}, z_{2}^{+}\right)-h\left(z_{1}^{+}, z_{2}\right) \leq 0
$$

because the function $h$ is decreasing with respect to the second variable and $z_{2}^{+} \geq z_{2}$. The derivative is therefore nonnegative, and therefore for $t_{1}>t_{0}, z_{1}\left(t_{1}\right) \leq z_{1}^{+}\left(t_{1}\right)$. Finally, we obtain the inequalities. All these arguments are classical in the field of monotone systems and differential inequalities [15].

Moreover, the same proof as the one of Lemma 2 proves that the maximal error vector $e=z^{+}-z^{-}$ remains bounded.

\subsection{Bundle of interval observers}

We have obtained guaranteed bounds on the estimation of the $z$, given bounds on the inputs. This is interesting by itself, but in the next paragraph we consider simultaneously several interval observers (with different couples $(\lambda, \theta)$ ), and, because we obtain guaranteed bounds for each observer, we can take their intersection, i.e. take the smaller envelope of the set of estimations. The idea is that, in this set of observers, some are going to be very good at the beginning of the estimations (for the transitories), other will give a better final error, ... Let us remark that, in this approach, any observer can only improve the smaller envelope of estimation, if it is an interval observer. Such an approach is not possible with classical observers (even if the inputs were perfectly known) because there is no notions of lower and upper estimates, coming here from the hypotheses of monotony (differential inequalities) and of the initial estimation $z^{-}(0) \leq z(0) \leq z^{+}(0)$. 


\begin{tabular}{|l|c|c|c|c|c|c|c|}
\hline Parameter & $c_{1}$ & $c_{2}$ & $S_{i n}$ & $P_{i n}$ & $a_{1}$ & $a_{2}$ & $a_{3}$ \\
\hline Value & 0.5 & 2 & $50+25 \cos (t / 5)$ & 5 & 1.2 & 0.5 & 15 \\
\hline Units & $/$ & $/$ & $\mathrm{g} / 1$ & $\mathrm{~g} / 1$ & $d a y^{-1}$ & $\mathrm{~g} / 1$ & $\mathrm{~g} / 1$ \\
\hline
\end{tabular}

Table 1: Parameters value used for the simulation of model (59).

Proposition 5 Let us fix the initial conditions $z^{-}(0) \leq z(0) \leq z^{+}(0)$, and consider a set (bundle) of observers for several couples $(\lambda, \theta)$ ) (including $\theta=0$ ) whose signs verify the condition of Proposition 1. Then the lower envelope $\bar{z}$ of the upper estimates $z^{+}$, and the upper envelope $\underline{z}$ of the lower estimates $z^{-}$is also a (better) guaranteed estimation of $z$, including and improving the open loop estimation.

The example in the next section will illustrate the proposition. Theoretical work remains to be done, of course, to obtain more analytical results, if possible.

\subsection{Bundle of interval observers with reinitialization}

As it will be made clear from the simulations below, in many cases it is advantageous to reinitialize the interval observers at regular times, because it improves still the smallest envelope of the estimates. It means that, at regular times, we restart the whole bundle of observers from the best estimation of the state at this time. The advantages of reinitialize the observers is well known [6].

\section{$6 \quad$ Study of an example}

To illustrate our methodology on a nontrivial example we take the model for denitrification presented throughout the paper. For the simulation purpose, we need an analytical expression for the bacterial growth rate. This growth rate (which is of course supposed to be not known) is a saturated function of $\mathrm{NO}_{3}$ and is inhibited by $\mathrm{NO}_{2}$ (nitrite is known to be toxic for growth). This leads to the following system:

$$
\left\{\begin{array}{l}
\dot{X}=a_{1} \frac{S}{S+a_{2}} \frac{a_{3}}{a_{3}+P} X-D X \\
\dot{S}=-c_{1} a_{1} \frac{S}{S+a_{2}} \frac{a_{3}}{a_{3}+P} X+D\left(S_{i n}-S\right) \\
\dot{P}=c_{2} a_{1} \frac{S}{S+a_{2}} \frac{a_{3}}{a_{3}+P} X+D\left(P_{i n}-P\right)
\end{array}\right.
$$

The values for the parameters can be found in Table 1, the evolution of $D(t)$ and $S_{i n}(t)$ are represented on Fig. 1.

We assume now that the available outputs are the biomass $\left(y_{1}=X\right)$ and the percentage of substrate in the medium:

$$
y_{3}=\frac{S}{S+P}
$$

As we have already seen, $y_{3}$ is a monotone function of $S$ and $P$. However, for negative values of $\hat{S}$, it becomes increasing with respect to $\hat{P}$, and for negative values of $\hat{P}$, it is a decreasing function of $\hat{S}$. Therefore we have to extend $h$ in the negative domain (for the observer). From the equation of $P$ in (59), since $P_{\text {in }}>0$ it is clear that there exists a positive $\epsilon$ such that $P(t)>\epsilon$ for all positive time (provided that $P(0)>\epsilon$ ).

We propose the following prolongation $\bar{h}(\hat{S}, \hat{P})$ : 
. If $\hat{S}<0$, then $\bar{h}(\hat{S}, \hat{P})=0$

. If $\hat{S} \geq 0$ and $\hat{P}<\epsilon$, then $\bar{h}(\hat{S}, \hat{P})=\frac{\hat{S}}{\hat{S}+\epsilon}$

. If $\hat{S} \geq 0$ and $\hat{P} \geq \epsilon$, then $\bar{h}(\hat{S}, \hat{P})=h(\hat{S}, \hat{P})$

It is easy to verify that $\bar{h}$ coincides with $h$ on $\{\hat{S} \geq 0, \hat{P} \geq \epsilon\}$ and has the same trend on $\mathbf{R}^{p}$ as $h$ on $\mathbf{R}_{+}^{p}$.

The initial conditions for $S$ and $P$ are respectively assumed to lie in the intervals [ $\left[\begin{array}{ll}0 & 20\end{array}\right]$ and $\left[\begin{array}{ll}0 & 30\end{array}\right]$.

Moreover, we know bounds for the influent concentrations: $S_{i n} \in[45+25 \cos (t / 5) 55+25 \cos (t / 5)]$ and $P_{\text {in }} \in\left[\begin{array}{ll}4.5 & 5.5\end{array}\right]$.

Now we can design the nonlinear observer proposed in Section 5 for system (59).

$$
\left\{\begin{array}{l}
\dot{z}_{1}^{+}=D\left(z_{i n 1}^{+}-z_{1}^{+}\right)-\theta \lambda_{1}\left(\bar{h}\left(S^{+}, P^{+}\right)-y_{3}\right) \\
\dot{z}_{2}^{+}=D\left(z_{i n 2}^{+}-z_{2}^{+}\right)-\theta \lambda_{2}\left(\bar{h}\left(S^{+}, P^{+}\right)-y_{3}\right) \\
z_{1}^{-}=D\left(z_{i n 1}^{-}-z_{1}^{-}\right)-\theta \lambda_{1}\left(\bar{h}\left(S^{-}, P^{-}\right)-y_{3}\right) \\
\dot{z}_{2}^{-}=D\left(z_{i n 2}^{-}-z_{2}^{-}\right)-\theta \lambda_{2}\left(\bar{h}\left(S^{-}, P^{-}\right)-y_{3}\right) \\
S^{+}=z_{1}^{+}-\frac{y_{1}}{c_{1}} \\
P^{+}=z_{2}^{+}+\frac{y_{1}}{c_{2}} \\
S^{-}=z_{1}^{-}-\frac{y_{1}}{c_{1}} \\
P^{-}=z_{2}^{-}+\frac{y_{1}}{c_{2}}
\end{array}\right.
$$

To guaranty the convergence of this observer, we need (according to Proposition 1) $\lambda_{1} \geq 0$ and $\lambda_{2} \leq 0$.

We have run such observers for 25 values of $\lambda$, and we have also run the simple asymptotic interval observer (corresponding to $\lambda=0$ ).

The results of these observers are presented on Figure 2. As it can be seen, the gain accelerates the converging rate of some observers in the transitory phase and for some of the observers it reduces the asymptotic error. As a consequence, the best estimate given by the lower envelope of the upper and lower observers bundle can be seen on Figure 3. It is worth noting that the estimates of $S$ is strongly improved in the sense that the uncertainty interval is drastically narrowed compared to the open loop observer. The estimation of $P$ converges much more rapidly.

Now Figure 4 presents the results obtained after reinitializing all the observers by the smallest estimated interval. At the beginning the reinitialization was very frequent (each 0.3 hour) and then the initialization frequency is decreased. The best estimate given by the envelope of the upper and lower observers network is presented on Figure 5. The reinitialization process contributes to strongly increase the converging rate for both variables, and obviously the steady-state interval estimate is unchanged.

\section{Conclusion}

These results illustrate the robust properties of these nonlinear observers that does not nedd the knowledge of all the model (the kinetics terms are seen as an unknown input) and which can deal with parametric uncertainties. We insist on the fact that the convergence properties presented here are global. The uncertainties that have been considered in this paper are very large, but they are realistic for biotechnological processes.

$\mathrm{RR} \mathrm{n}^{\circ} 4905$ 

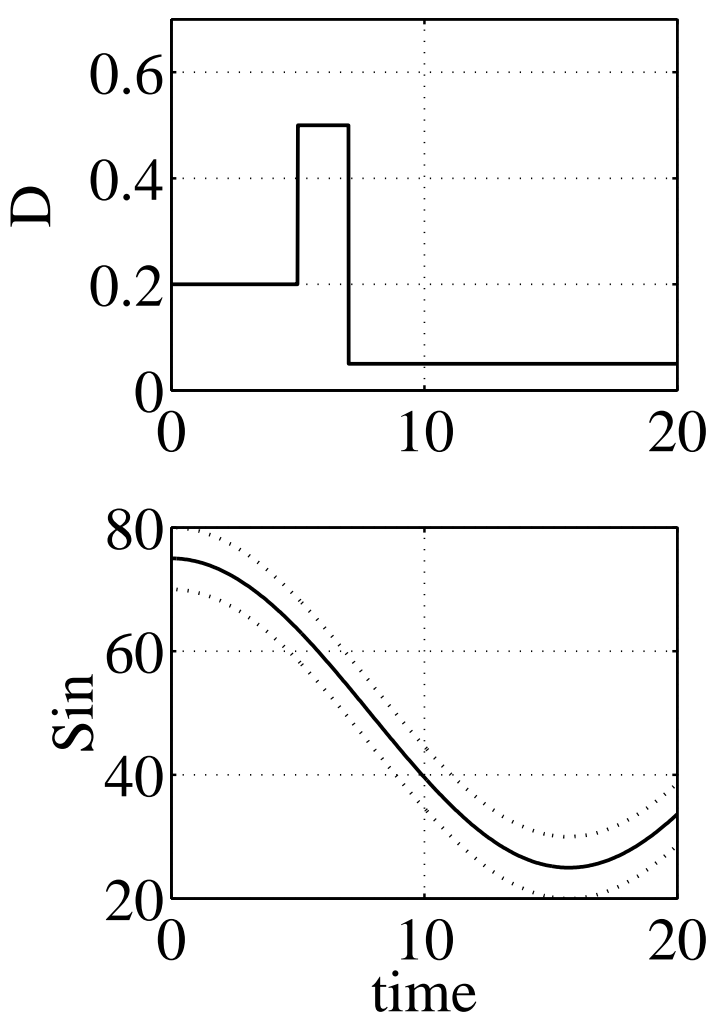

Figure 1: The inputs $D$ and $S_{i n}$. 

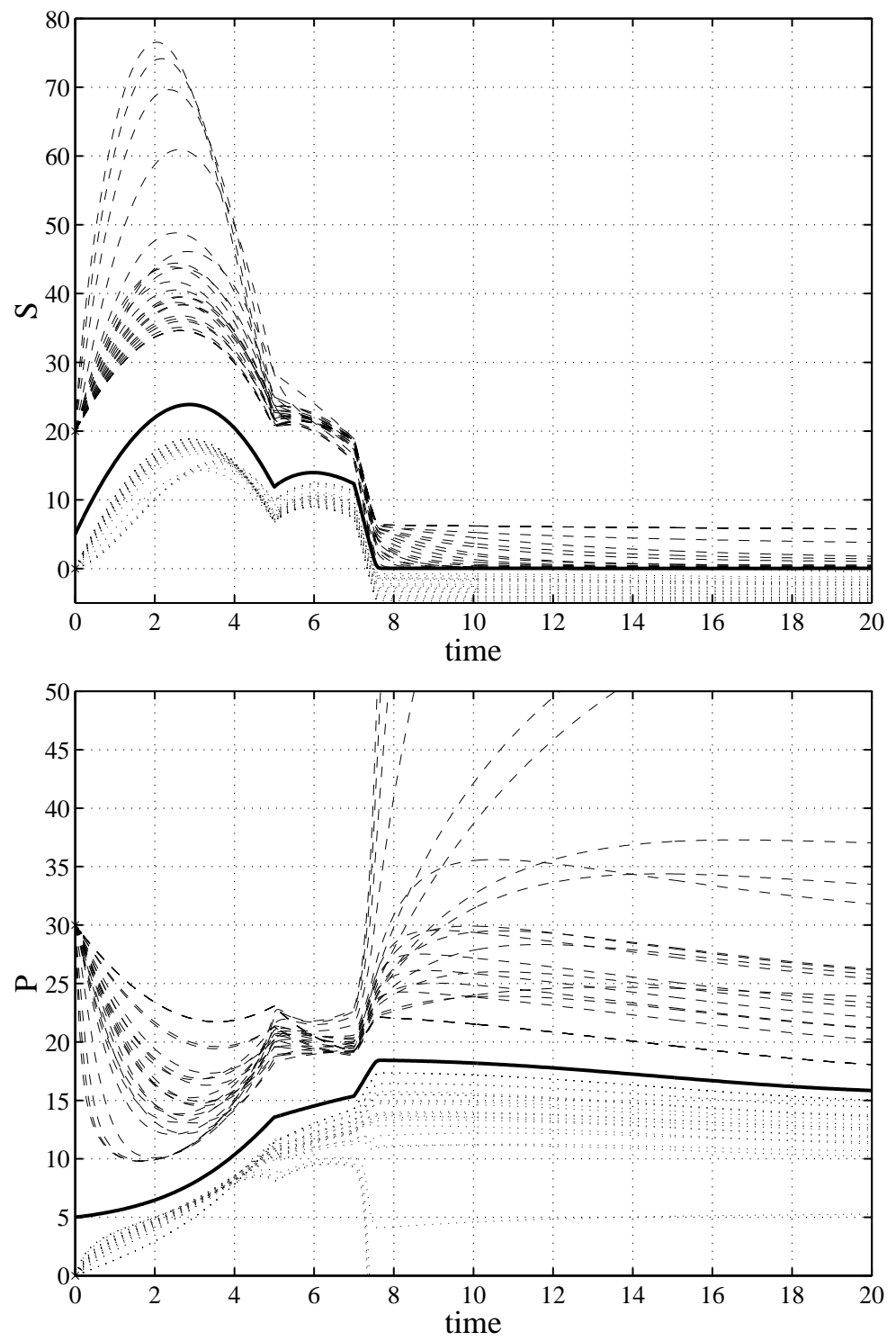

Figure 2: Set of observers for bounded uncertainties in $S_{i n}$ and $P_{i n}$. The upper values are dashed and the lower value are dotted. The actual values are in continuous lines. 

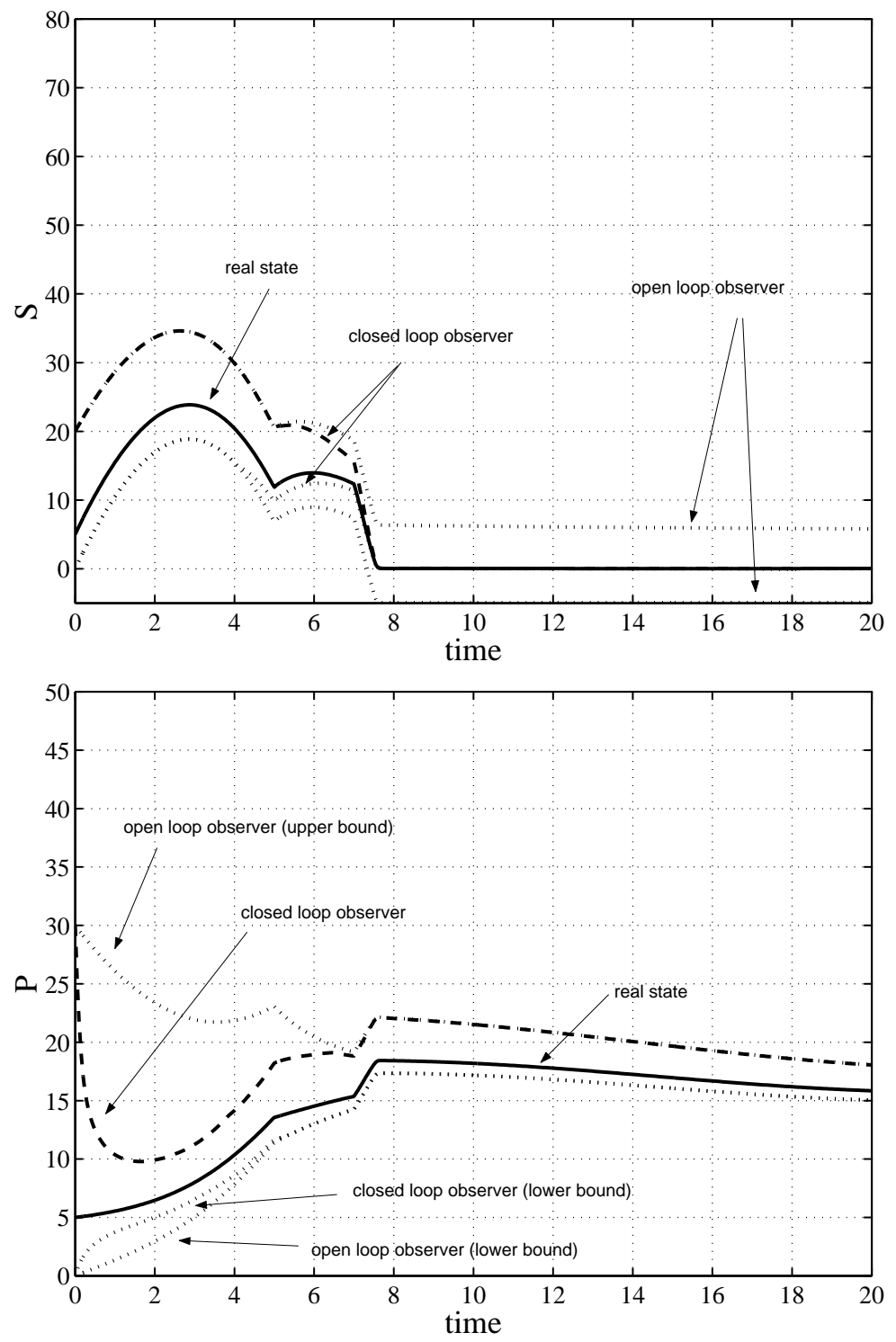

Figure 3: Lower envelope of the estimates given by the set of observers, for uncertainties in $S_{i n}$ and $P_{i n}$. The actual values are in continuous lines. The estimate of the open loop interval observer is plotted in dots. 

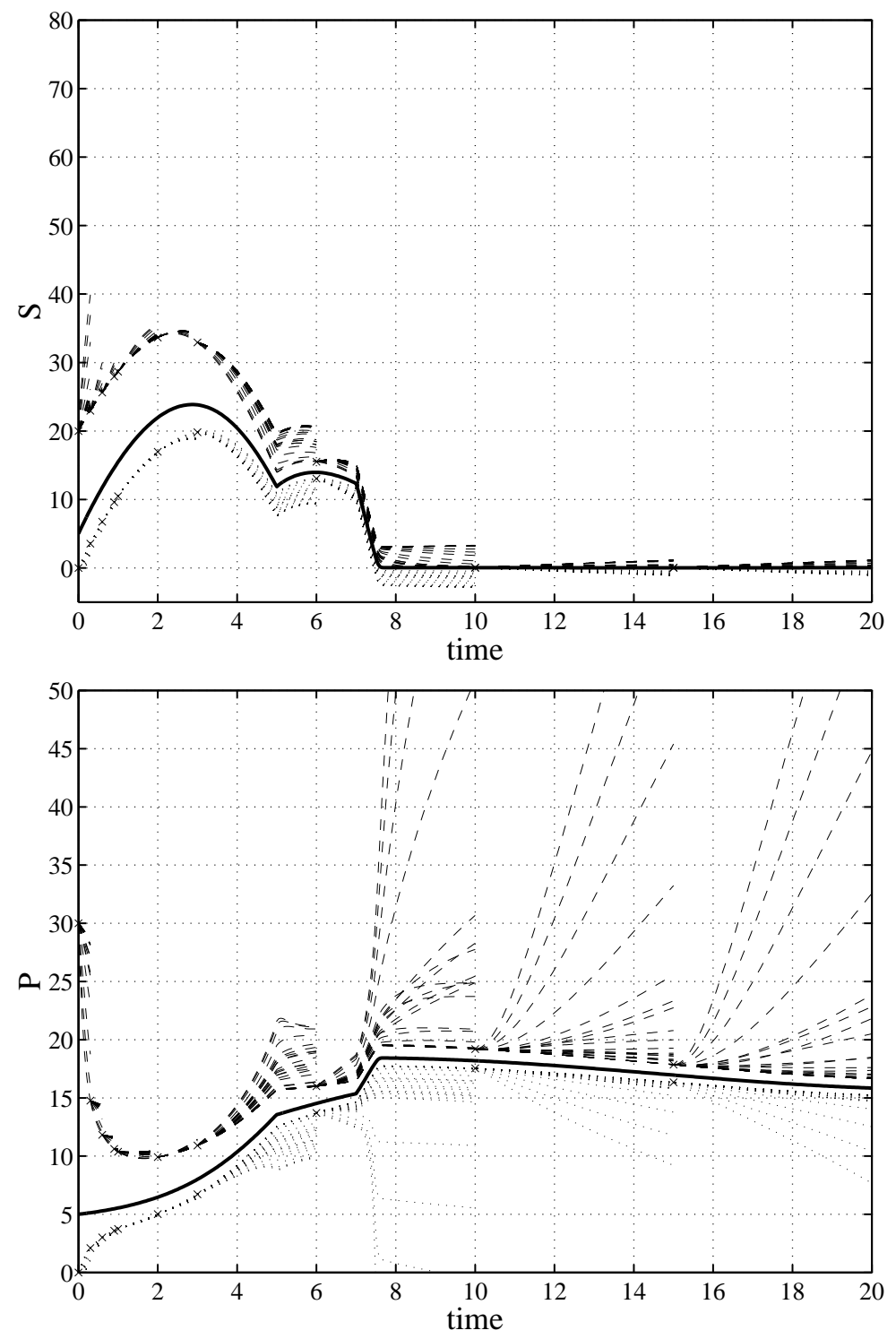

Figure 4: Set of observers for bounded uncertainties in $S_{i n}$ and $P_{i n}$. The observers are reinitialized to improve the global convergence rate of the envelope. The upper values are dashed and the lower value are dotted. The actual values are in continuous lines. 

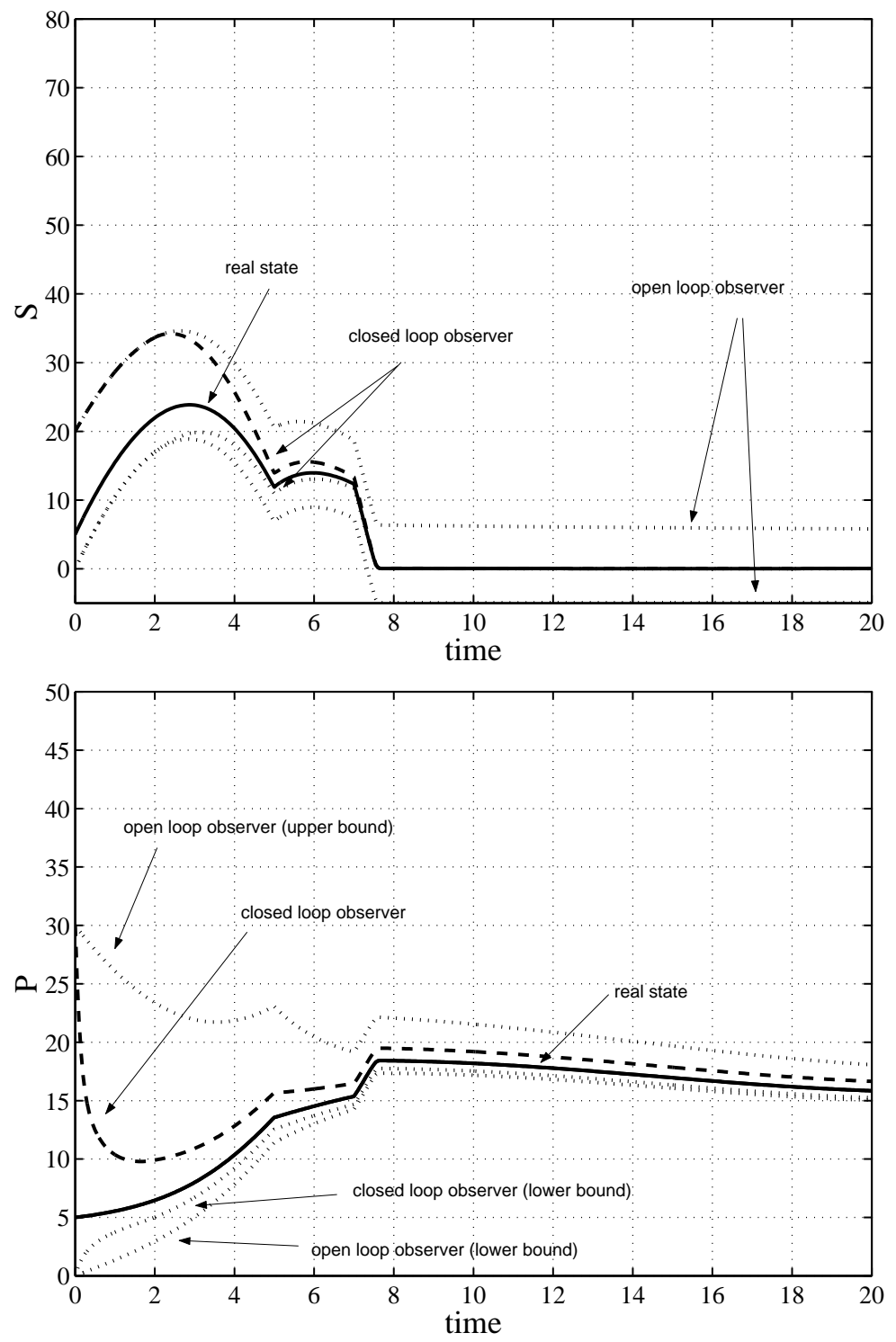

Figure 5: Envelope of the estimates given by the set of reinitialized observers, for uncertainties in $S_{\text {in }}$ and $P_{i n}$. The actual values are in continuous lines. The estimate of the open loop interval observer is plotted in dots. 
Note that the converging rate of each of these observers can generally not be tuned. It turns out that the slowest eigenvalue is always $D$. By considering a bundle of observers, we are nevertheless able to improve the rate of convergence and the asymptotic bounds. Of course, additional theoretical work is to be done.

The same principle applies for uncertainties in the yield coefficients or bounded noise on the outputs.

Acknowledgments: The authors wish to thank the two reviewers for their attentive readings and pertinent suggestions. They acknowledge the support of the action RTP50 / STIC of CNRS.

This work has been carrying out with the support provided by the European commission, Information Society Technologies program, Key action I Systems \& Services for the Citizen, contract TELEMAC number IST-2000- 28256. This information is provided under the sole responsibility of the author and does not necessarily represent the opinion of the European Commission, which is not responsible for any use that might be made of it.

\section{References}

[1] G. Bastin and D. Dochain. On-line estimation and adaptive control of bioreactors. Elsevier, 1990.

[2] G. Bastin and J. VanImpe. Nonlinear and adaptive control in biotechnology: a tutorial. European Journal of Control, 1(1):1-37, 1995.

[3] O. Bernard. Mass balance modelling of bioprocesses. In A.Agrachev, editor, Mathematical control theory, pages 769-812. ICTP lecture notes, 2002.

[4] O. Bernard, Z. Hadj-Sadok, and J.-L. Gouzé. Observers for the biotechnological processes with unknown kinetics. application to wastewater treatment. In Proceedings of CDC 2000. Sydney, Australia, 2000.

[5] O. Bernard, G. Sallet, and A. Sciandra. Nonlinear observers for a class of biological systems. Application to validation of a phytoplanktonic growth model. IEEE Trans. Autom. Contr., 43:1056-1065, 1998.

[6] E. Busvelle and J. Gauthier. High-gain and non-high-gain observers for nonlinear systems. In AnzaldoMeneses, A., Bonnard, B.Gauthier, J.P. ; Monroy-Pérez, F., editor, Contemporary trends in nonlinear geometric control theory and its applications. International conference, México City, México. World Scientific, 2002.

[7] M. Darouach, M. Zasadzinski, and S.-J. Xu. Full-order observers for linear systems with unknown inputs. IEEE Trans. Autom. Cont., 39(3):1068-1072, 1994.

[8] F. Deza, E. Busvelle, J. Gauthier, and D. Rakotopara. High gain estimation for nonlinear systems. System and control letters, 18:292-299, 1992.

[9] J. P. Gauthier, H. Hammouri, and S. Othman. A simple observer for nonlinear systems applications to bioreactors. IEEE Trans. Autom. Contr., 37:875-880, 1992.

[10] J. L. Gouzé, A. Rapaport, and Z. Hadj-Sadok. Interval observers for uncertain biological systems. Ecological modelling, 133:45-56, 2000.

$\mathrm{RR} \mathrm{n}^{\circ} 4905$ 
[11] M. Z. Hadj-Sadok and J. L. Gouzé. Estimation of uncertain models of activated sludge process with interval observers. Journal of Process Control, 11(3):299-310, 2001.

[12] M. Hou and P. Müller. Design of observers for linear systems with unknown inputs. IEEE Trans. Autom. Contr., AC-37(6):871-875, 1991.

[13] P. Kudva, N. Viswanadham, and A. Ramakrishna. Observers for linear systems with unknown inputs. IEEE Trans. Autom. Contr., AC-25(1):113-115, 1980.

[14] A. Rapaport and J.-L. Gouzé. Parallelotopic and practical observers for nonlinear uncertain systems. Int. Journal. Control, 76(3):237-251, 2003.

[15] H. L. Smith. Systems of ordinary differential equations which generates an order preserving flow : A survey of results. SIAM Review, 30:87-113, 1988.

[16] M. Vidyasagar. Nonlinear Systems Analysis. Prentice-Hall, Englewood Cliffs, New Jersey, 1993. 


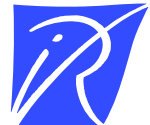

Unité de recherche INRIA Sophia Antipolis

2004, route des Lucioles - BP 93 - 06902 Sophia Antipolis Cedex (France)

Unité de recherche INRIA Futurs : Parc Club Orsay Université - ZAC des Vignes

4, rue Jacques Monod - 91893 ORSAY Cedex (France)

Unité de recherche INRIA Lorraine : LORIA, Technopôle de Nancy-Brabois - Campus scientifique

615, rue du Jardin Botanique - BP 101 - 54602 Villers-lès-Nancy Cedex (France)

Unité de recherche INRIA Rennes : IRISA, Campus universitaire de Beaulieu - 35042 Rennes Cedex (France)

Unité de recherche INRIA Rhône-Alpes : 655, avenue de 1'Europe - 38334 Montbonnot Saint-Ismier (France)

Unité de recherche INRIA Rocquencourt : Domaine de Voluceau - Rocquencourt - BP 105 - 78153 Le Chesnay Cedex (France)

Éditeur

INRIA - Domaine de Voluceau - Rocquencourt, BP 105 - 78153 Le Chesnay Cedex (France)

http://www.inria.fr

ISSN 0249-6399 